\title{
Structural change and nucleation characteristics of water/ice in confined geometry
}

\author{
John Dore, Beau Webber \\ [Physics Lab, University of Kent, Canterbury CT2 7NR, UK] \\ Dan Montague \\ [ex-Willamette University, Oregon, USA] \\ Thomas Hansen \\ [Institut Laue-Langevin, F 38042 Grenoble Cedex 9, France]
}

Contact author: J.C.Dore@ukc.ac.uk

\section{Introduction}

The confinement of liquids in the restricted space of a mesoporous solid leads to significant changes in their properties. The main effect is a reduction in the nucleation temperature that, according to the Gibbs-Thomson formalism is inversely proportional to the pore size. Other effects on the liquid structure and transport properties also occur and depend on the detailed characteristics of the mesoporous material. Confined liquids occur in various situations, both in the natural environment and also in industrial processes, so that there is a growing interest in understanding the fundamental principles that govern the behaviour and modified characteristics.

For scientific study it is important to have a well-characterised system in which the solid matrix has a large pore volume, controlled narrow pore size distribution function and high surface area. The most convenient material for this purpose is silica and there is a wide range of pore sizes available that can be produced by the sol-gel process. More recently, other forms of mesoporous silica such as MCM and SBA-types have been fabricated with an ordered arrangement of cylindricallyshaped pores made by a template process. The internal silica surface is normally smooth and hydrophilic so that surface wetting is not usually a problem and the water is easily adsorbed into the pore volume. In contrast, the activated carbons, which are also extremely important in industrial use, have a much more complex pore and surface structure which makes a detailed interpretation of the experimental measurements more difficult. Consequently, the main interest has focused on silicas as the ideal substrate and the initial investigation of liquids in confined geometry has been centred on these materials.

\section{Theoretical background}

The formalism for neutron scattering by molecular liquids has been presented elsewhere [1,2] and only a brief summary will be given here. The measured diffraction pattern can be converted into a molecular structure factor, $S_{M}(Q)$, which may be divided into intra- and inter-molecular contributions, i.e.

$$
\mathrm{S}_{\mathrm{M}}(\mathrm{Q})=\mathrm{f}_{1}(\mathrm{Q})+\mathrm{D}_{\mathrm{M}}(\mathrm{Q})
$$

where $f_{l}(Q)$ is the molecular form factor and $D_{M}(Q)$ defines the spatial relationship between the molecules. The real-space distribution function $\mathrm{d}_{\mathrm{L}}(\mathrm{r})$ can be obtained from the Fourier-Bessel transform:- 


$$
\mathrm{d}_{\mathrm{L}}(\mathrm{r})=4 \pi \mathrm{r} \rho_{\mathrm{M}}[\overline{\mathrm{g}}(\mathrm{r})-1]=\frac{2}{\pi} \int_{0}^{\infty} \mathrm{QD}_{\mathrm{M}}(\mathrm{Q}) \sin \mathrm{Qr} \mathrm{dQ}
$$

where $\rho_{M}$ is the molecular number density and $\bar{g}(r)$ is a composite pair correlation function, which for $\mathrm{D}_{2} \mathrm{O}$ is :-

$$
\overline{\mathrm{g}}(\mathrm{r})=0.092 \mathrm{~g}_{\mathrm{OO}}(\mathrm{r})+0.423 \mathrm{~g}_{\mathrm{OD}}(\mathrm{r})+0.485 \mathrm{~g}_{\mathrm{DD}}(\mathrm{r})
$$

The measurement for liquids confined in the voids of a solid porous substrate requires the subtraction of the substrate scattering from the total scattering by the 'liquid + substrate'. This method needs careful adjustment of the relative intensities to allow for the variable attenuation factors. The direct analysis also assumes that the interference contributions resulting from the liquid-substrate cross-term are negligible and this factor depends on the pore size, which influences the relative contributions. This approximation is satisfactory for pore sizes of $>50 \AA$ but is inadequate at lower values. The dispersed nature of the liquid also leads to an effective broadening of the diffraction profile if the pore dimensions are small. This phenomenon is similar to that occurring in the diffraction broadening of Bragg peaks for small crystallite sizes and is a result of the limitations in the volume integral which leads to an effective convolution of the full pattern with a function $M(Q)$, that is dependent on the pore size distribution [3]. In the case of liquids, the overall shape of the diffraction pattern does not have sharp features and the diffraction broadening effect is significant only for small pores $(<20 \AA)$.

Another method of analysis is to use a first-order difference function to analyse the structural changes with temperature. This technique has a number of advantages, particularly for studies in confined geometry, since it eliminates the substrate and intra-molecular scattering in a systematic manner. The temperature difference function is defined by

$$
\Delta \mathrm{D}_{\mathrm{M}}(\mathrm{Q}, \Delta \mathrm{T})=\mathrm{S}_{\mathrm{M}}(\mathrm{Q}, \mathrm{T})-\mathrm{S}_{\mathrm{M}}\left(\mathrm{Q}, \mathrm{T}_{\mathrm{o}}\right)
$$

in relation to a chosen reference temperature, $\mathrm{T}_{\mathrm{o}}$ with $\Delta \mathrm{T}=\left|\mathrm{T}-\mathrm{T}_{0}\right|$ and the corresponding transform yields the real-space function

$$
\Delta \mathrm{d}_{\mathrm{L}}(\mathrm{r}, \Delta \mathrm{T})=\frac{2}{\pi} \int_{\mathrm{O}}^{\infty} \mathrm{Q} \Delta \mathrm{D}_{\mathrm{M}}(\mathrm{Q}, \Delta \mathrm{T}) \sin \mathrm{Qr} \mathrm{dQ},
$$

which defines the structural re-arrangement. The low r-value behaviour of $d_{L}(r)$, where $g(r)$ is zero, gives the microscopic density through the relation

$$
d_{L}(r)=-\left(4 \pi \rho_{M}\right) r
$$

and the corresponding expression for $\Delta \mathrm{d}_{\mathrm{L}}(\mathrm{r})$ is

$$
\Delta \mathrm{d}_{\mathrm{L}}(\mathrm{r})=-\left(4 \pi \Delta \rho_{\mathrm{M}}\right) \mathrm{r},
$$

which depends on the change in density, $\Delta \rho_{M}(\Delta T)$. 


\section{Review of earlier results}

\section{a) Super-cooled water in sol-gel silicas}

The first neutron diffraction studies of water in a series of commercial sol-gel silicas $[3,4]$ were reported in the early 1980's and were followed by further studies covering different pore sizes which have been reviewed comprehensively [5] for work done up to 2000. Normally, super-cooled water would be metastable at these temperatures but the effect of the confinement means that the liquid phase is in an equilibrium state and forms readily under these conditions. The neutron results confirmed the depression of nucleation point, which had been observed by other techniques, and provided information on the changing structure of the water network. The main features are illustrated in Figs 1 and 2, which show the variation in the diffraction pattern for $\mathrm{D}_{2} \mathrm{O}$ water with temperature and the shift of the main diffraction peak position as a function of temperature, $Q_{0}(T)$, for several mesoporous silicas of varying pore size. The peak profile in Fig 1 changes systematically and indicates the development of a shoulder on the high-Q side as the temperature is reduced. The variation of the peak position shown as a solid line in Fig $2 \mathrm{a}$ is monotonic across the whole temperature range but shows a systematic increase in slope below the point of maximum density, which is $11^{\circ} \mathrm{C}$ for $\mathrm{D}_{2} \mathrm{O}$. The trend is towards a value of $1.7 \AA^{-1}$ at a temperature of $-40^{\circ} \mathrm{C}$, which corresponds to the limiting case of homogeneous nucleation of $\mathrm{D}_{2} \mathrm{O}$ at ambient pressure [6]. The confinement of water in pores gives a displacement of the peak position which may be attributed to enhanced hydrogen-bonding and is shown as single points for a range of silicas with different pores sizes.

Most molecular liquids would have a very small change in the $\mathrm{Q}_{0}$ value arising simply from density changes but water exhibits a much larger effect that can be linked to the extended formation of the hydrogen-bonded network. The studies of deeply super-cooled water in the bulk phase have demonstrated the enhancement of the intermediate-range correlations as the temperature is reduced, as shown in Fig 2b. The picture to emerge from these studies is of an evolution towards the extended continuous random network of tetrahedral hydrogen-bonds that characterises the structure of low-density amorphous ice [7]. The overall behaviour of the confined water is therefore similar to that of bulk water, except that the hydrogen-bonded network appears to be more developed relative to that of bulk water at the same temperature, as shown by the data in Fig 2.

\section{b) Water and ice in MCM silicas}

MCM silicas consist of two main types with cylindrical pores of 30-35 Å diameter; MCM41 is based on straight parallel channels arranged in an approximately hexagonal lattice, whereas MCM48 is formed from a cubic liquid crystal phase which has branched channels of a similar diameter. Neutron diffraction measurements of $\mathrm{D}_{2} \mathrm{O}$ water in both of these materials have been made and show that deep super-cooling occurs in which the liquid phase may exist at $45^{\circ} \mathrm{C}$ below the normal freezing point $\left[5^{\circ} \mathrm{C}\right.$ for $\left.\mathrm{D}_{2} \mathrm{O}\right]$. The diffraction pattern undergoes a systematic change as the temperature is lowered, as shown in Fig 3a. The temperature difference function, $\Delta \mathrm{D}_{\mathrm{M}}(\mathrm{Q}, \Delta \mathrm{T})$, can be evaluated 


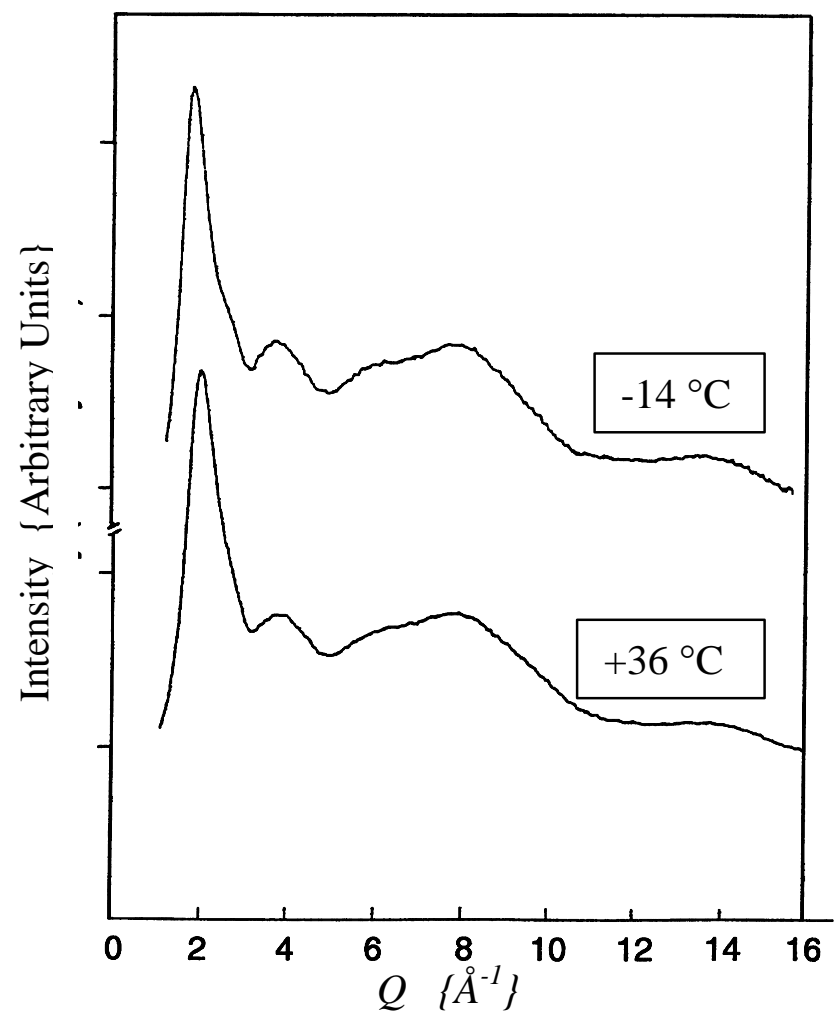

Figure 1. The change in the diffraction pattern for $\mathrm{D}_{2} \mathrm{O}$ with temperature
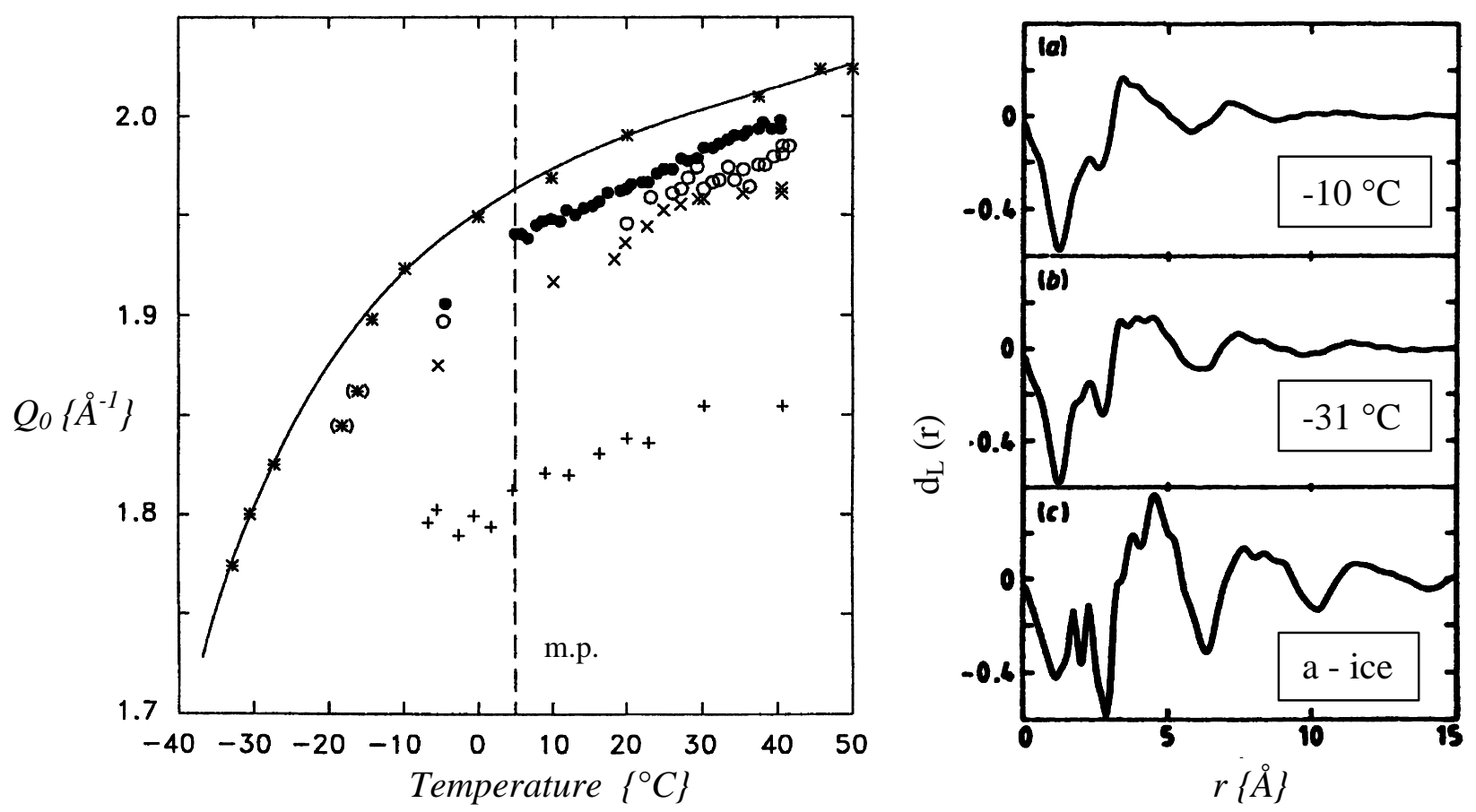

Figure 2. Super-cooled water a) variation of $\mathrm{Q}_{0}(\mathrm{~T})$ for bulk and confined water in various sol-gel silicas, b) change of $\mathrm{d}_{1}(\mathrm{r})$, with temperature $\left[-10{ }^{\circ} \mathrm{C},-31^{\circ} \mathrm{C}\right.$ and amorphous ice]. 
from these datasets and transformed to give the $\Delta d_{L}(r, \Delta T)$ function shown in Fig 3b. It is clear that these results are similar to those seen in the bulk phase behaviour but extend to much lower temperatures and show the continued displacement of the peak $\mathrm{Q}_{0}(\mathrm{~T})$ towards the position for hexagonal ice; the profile also sharpens due to the presence of longer-range correlations. The spatial distribution function also shows an increase in the correlations over the intermediate range ( $5 \AA<\mathrm{r}<15 \AA$ ) corresponding to a growth of the hydrogen-bond network, as expected. Another unexpected finding was that the transition was completely reversible and showed no evidence of the hysteresis effects that usually occur for nucleation and melting in confined geometry; this feature is discussed further in Sec 3e..

The reasons for the formation of the defective cubic ice are still not understood but it seems to be reproduced in all studies involving the formation of small ice crystallites that are restricted in growth.

\section{c) Over-filled samples of water and ice in MCM silicas}

The neutron investigation is sensitive to the total amount of water in the sample so that excess water above the amount needed to fill the pore volume will reside on the outer surfaces of the particles or grains. On cooling, this external water will freeze before that inside the pores and, as shown in Fig 3a for an over-filled MCM48 sample, there is some super-cooling before nucleation to hexagonal ice at $249 \mathrm{~K}$, which is indicated by the triplet profile of the first diffraction peak. If the temperature is further reduced, the pore water freezes as cubic ice and the central peak of the triplet grows. However, the peak on the low$\mathrm{Q}$ side also increases in intensity showing that the defective form of cubic ice is again created, so there is no growth of the external ice with hexagonal form into the pore volume. Another feature shown in these curves is that even after nucleation, the diffraction pattern changes slightly as the temperature is further reduced to $206 \mathrm{~K}$, indicating that there are further structural changes after the main nucleation event has occurred. The additional Bragg peaks at higher Q-values also mimic these changes as shown in the difference functions. Similar results are shown for MCM41 measurements with an over-filled water sample.

A more detailed picture can be obtained by transforming the $\Delta \mathrm{D}_{\mathrm{M}}(\mathrm{Q}, \Delta \mathrm{T})$ function with a reference temperature of $\mathrm{T}_{0}=236 \mathrm{~K}$, to give a representation of the changes in real space after the hexagonal ice has formed and relative to the deeply super-cooled liquid at $\sim 45 \mathrm{~K}$ below its normal bulk freezing point. Fig.4a shows three sets of diffraction data for over-filled MCM48 silicas at temperatures of 226,216 and $206 \mathrm{~K}$; the corresponding $\Delta \mathrm{d}_{\mathrm{L}}(\mathrm{r}, \Delta \mathrm{T})$ curves are shown in Fig 4b. The absence of a peak at $1.0 \AA$ indicates that the molecular terms of the $\mathrm{f}_{1}(\mathrm{Q})$ form-factor have been accurately eliminated in the difference function analysis. The presence of sharp peaks at 1.8 and $2.1 \AA$ arises from local hydrogen-bonds in the ice lattice and suggests that there is a sharpening of the spatial correlations as the cubic ice forms. This feature presumably means that the H-bond angle variation becomes more restricted and consequently affects the longer-range correlations, which show a similar behaviour out to $20 \AA$. It therefore becomes clear that the deeply super-cooled water has considerable disorder relative to the defective form of cubic ice. Furthermore, there are additional changes in the $20 \mathrm{~K}$ region below the main nucleation event, as observed previously but less clearly in earlier studies. The development of deep minima at $6.5,10.3$ and $14 \AA$ is also a characteristic feature of the d(r) function for amorphous ice and seems to be a signature of long-range order in $\mathrm{H}$-bonded networks. However, there are also interesting features in the shape of the curves in the $4-10 \AA$ region that have not yet been fully interpreted. 

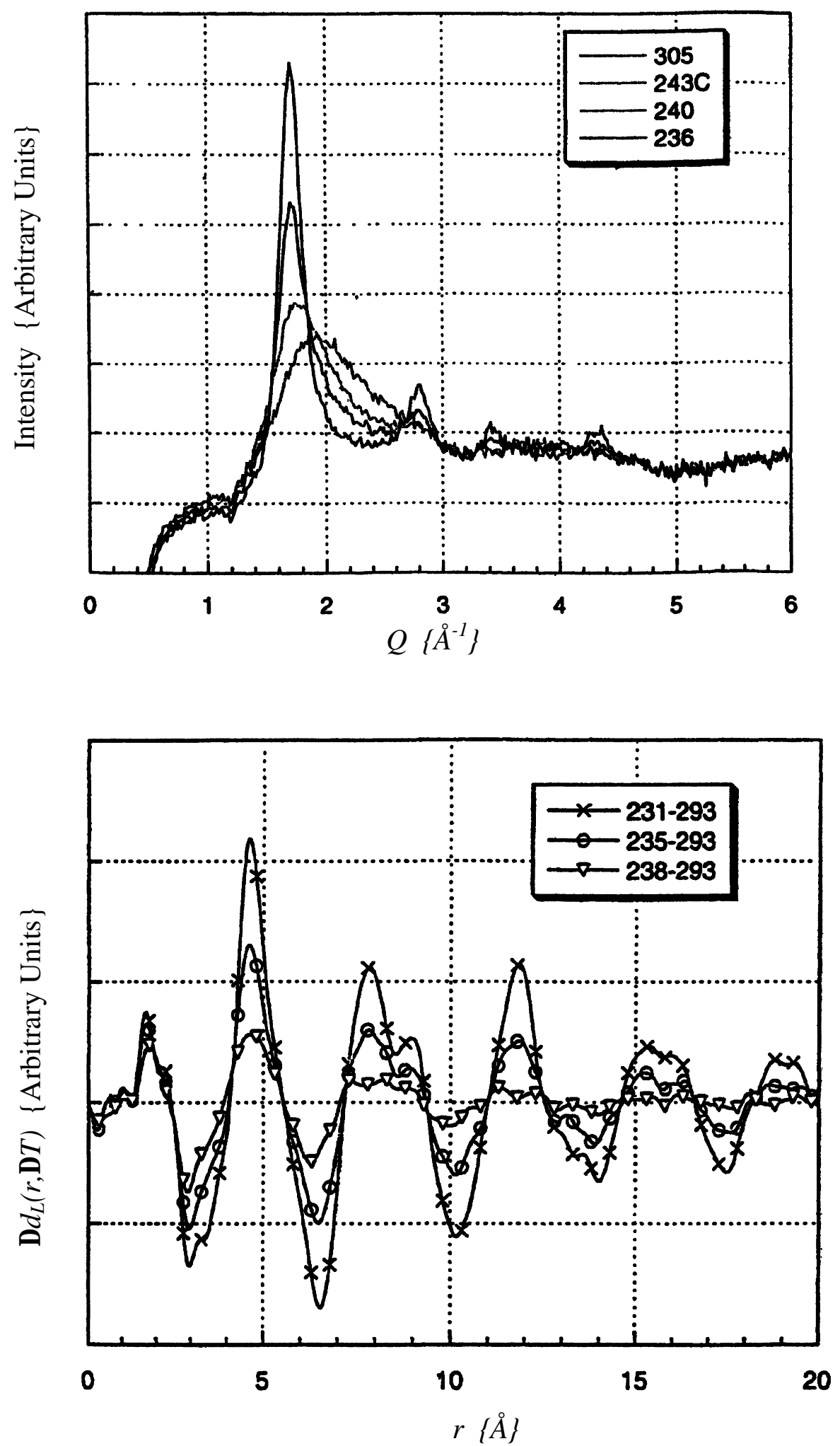

Figure 3. Neutron diffraction studies of a sample of $\mathrm{D}_{2} \mathrm{O}$ in MCM41 silica measured on the D4 diffractometer:

a) scattering intensity from water/ice as a function of temperature,

b) the structural change, $\Delta \mathrm{d}_{\mathrm{L}}(\mathrm{r}, \Delta \mathrm{T})$, for the same datasets. 

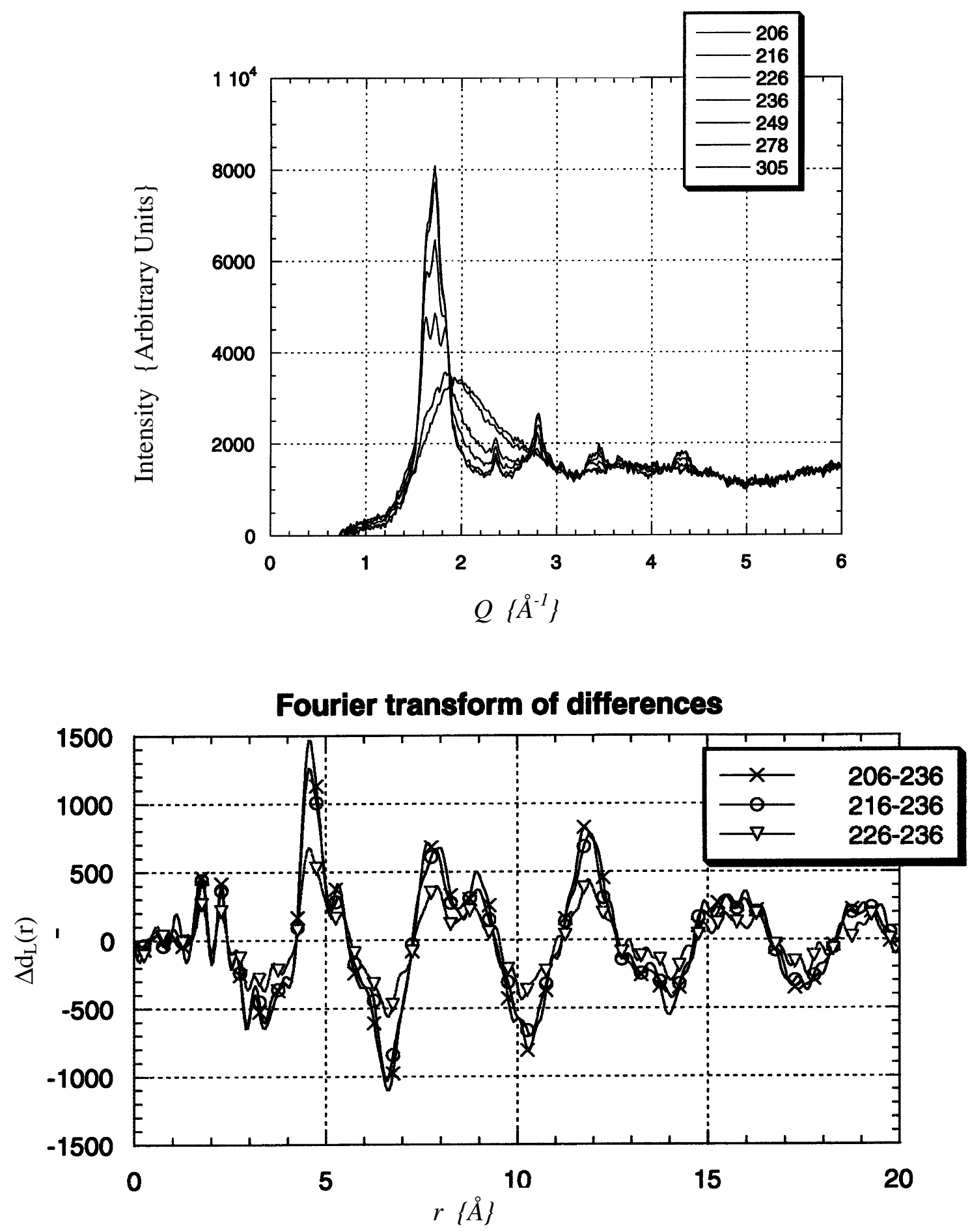

Figure 4. Neutron diffraction of $\mathrm{D}_{2} \mathrm{O}$ in an overfilled sample of MCM48 silica taken on the D20 diffractometer: a) scattering intensity from water/ice as a function of temperature, $b)$ the structural change, $\Delta d_{L}(r, \Delta T)$, for the same datasets with a reference temperature of $236 \mathrm{~K}$. 


\section{d) Ice nucleation in various sol-gel silicas}

A neutron diffraction study was made of nucleation in a range of sol-gel silicas of different sizes [8] where the effective water thickness was maintained at approximately $20 \AA$ by partial filling of the larger pore materials. The comparison of the primary ice peaks is shown in Fig 5. For the large pore size, the triplet that characterises hexagonal ice $\mathrm{I}_{\mathrm{c}}$ is clearly shown. However, the peak profiles for the ice formed in the materials of lower pore size show an asymmetric broadening of the peaks which is characteristic of facetted growth along preferred axes [9]. Cubic ice $\mathrm{I}_{\mathrm{c}}$ would display only a single peak at the central position, so it appears that there is a tendency towards a cubic ice structure, which is also revealed in the secondary peaks. However, pure cubic ice is not created in the pores and the structure must therefore be classified as 'defective cubic ice'. It has been suggested that this feature could arise from stacking faults as the basic six-membered rings are present in both forms of ice I but no quantitative treatment has been possible that provides an explanation of the observed peak profiles. In this, context it will be interesting to relate the findings to the simulation studies of nucleation in bulk phase water presented at this meeting; see also Sec 5.

\section{e) Ice nucleation in MCM silicas}

An unexpected finding in the study of ice nucleation and melting for water in MCM silicas was that the phase transition was completely reversible and showed no evidence of hysteresis effects that usually occur for nucleation and melting in confined geometry. The situation is well illustrated in Fig 6.which shows two superimposed plots of the measured intensity at the same stabilised temperature, just prior to nucleation in a cooling run and just after melting in a heating run. The difference function (multiplied by a factor of 10 for clarity) is shown beneath the two plots and indicates that the two curves are identical to within the statistical accuracy of the measurements. These results are now known to be in agreement with the comprehensive DSC studies of Findenegg and colleagues [10] but are not necessarily reproduced for larger pore sizes in the ordered mesoporous silicas of MCM or SBA-type. The nucleation event is itself of considerable interest and is discussed in the following section.

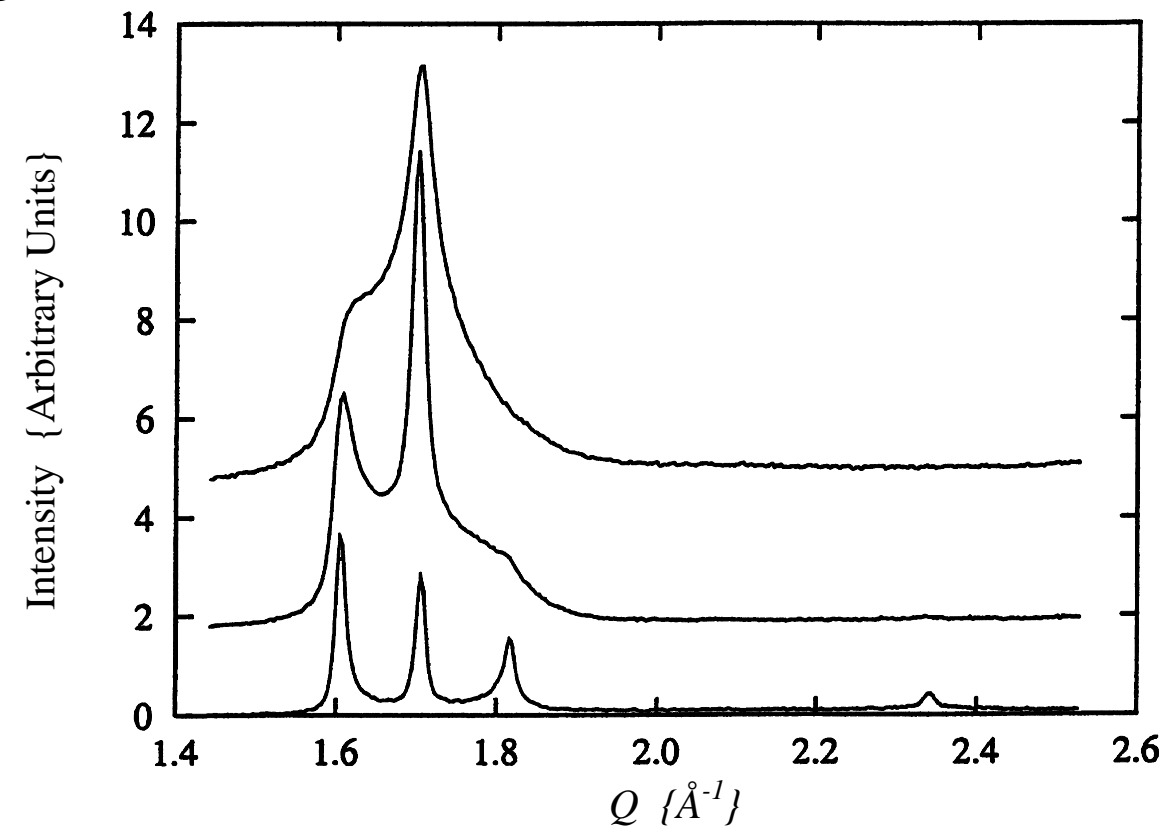

Figure 5. The first diffraction peak for ice nucleation in sol-gel silicas with different pore size; see the text for details. 


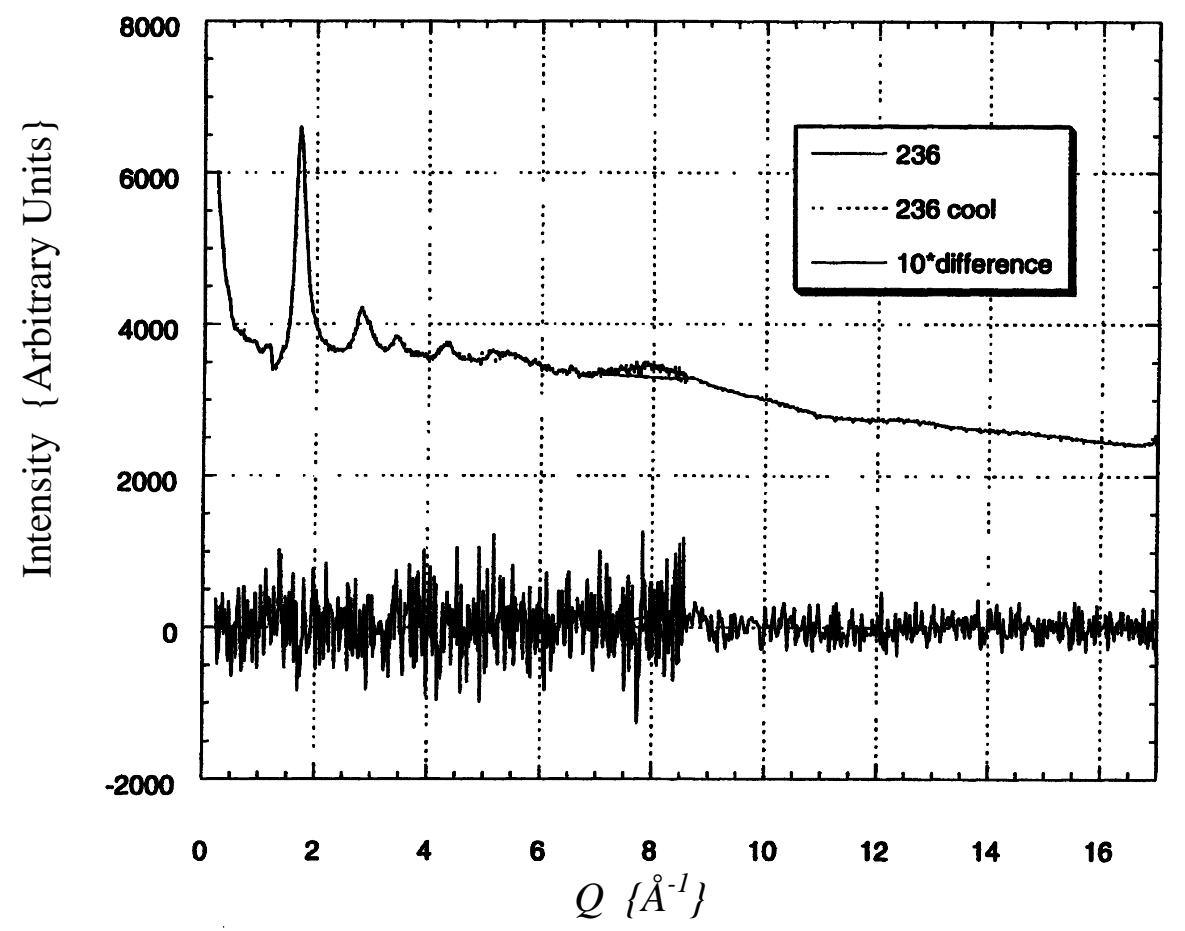

Figure 6. Two superimposed datasets for cooling and heating runs at a temperature of $236 \mathrm{~K}$ taken with the two multidetectors on the D4 diffractometer, showing the complete reversibility of the transition after the nucleation of cubic ice; the difference between the two datasets is shown below multiplied by a factor of $x 10$.

\section{f) The cubic ice phase}

The cubic ice phase has a similar local structure to that of hexagonal ice, consisting of sixmembered rings of hydrogen-bonded molecules. The structure factor has a single peak at $1.70 \AA^{-1}$, which is at the central peak position for the hexagonal ice triplet. High resolution studies of nucleation in several sol-gel silicas have revealed important changes in the peak profile. The hexagonal ice triplet has Bragg peaks at Q-values of $1.55,1.70$ and $1.85 \AA^{-1}$ corresponding to $(1,0,0),(0,0,2)$ and $(1,0,1)$ reflections. The results indicate that a defective form of cubic ice is formed in the smaller pores. There is also a secondary peak at $2.33 \AA^{-1}$ that is present for ice $I_{c}$ and not for ice $I_{h}$ so that the two phases are easily distinguished, in principle. However, all the diffraction observations give a broadened first peak with a shoulder on the low-Q side, or even a separated peak, if the Q-resolution is sufficient (Fig.5). It therefore seems that pure cubic ice is never formed even though there are several different routes to its production from either the liquid or the amorphous ice phase and also when produced by slow vapour-deposition at temperatures just above $145 \mathrm{~K}$. The reasons for this behaviour are still not understood but it seems to be reproduced in all studies involving the formation of small ice crystallites that are restricted in growth.

The most likely explanation of this phenomenon rests with the initial formation of a small hydrogen-bonded assembly that will act as a nucleus for crystal growth. When the protocrystallite is small the crystal axes will not be well defined and the subsequent initial growth will contain a number of stacking faults. As subsequent layers are added during the growth process, it seems that these defects become 'frozen in' but when the crystallite 
has grown to a certain size, the change in surface-to-volume factor causes a modification in molecular orientations and the crystal readily grows into the hexagonal form. Furthermore, it is well known that, for hexagonal ice, the growth in the basal plane is much faster than that in the perpendicular direction and this effect could possibly be a key to understanding the preference for the hexagonal form in larger crystals. Since defective cubic ice is observed for pore sizes up to $200 \AA$ but pure hexagonal ice is observe even for thin films in $500 \AA$ pores, there must be a critical size effect in the region of $300 \AA$ corresponding to a change in the growth process. In the case of the MCM silicas the situation is more complex as the cylindrical pore restricts growth in two directions but also permits growth along the pore axis. Current experimental work has been conducted for powdered samples so that the observed diffraction profile is an orientationally-averaged pattern. Since there appears to be little difference in the nucleation behaviour for MCM41 with straight pores and MCM48 with branched pores, it would seem likely that the constrained crystallites do not have a strongly-correlated orientation with respect to the pore axis. However, this conjecture cannot be checked at present because there are no ways of producing a silica monolith with well-defined pore size and axial orientation.

Another uncertainty concerns the density variation for the confined water/ice. It is assumed that the density of cubic ice is the same as that of hexagonal ice, which is $8 \%$ less than that of water at ambient temperatures but there is no easy means of determining the real variation in the density of the super-cooled water in the mesopores. It is convenient to assume that the changes correspond to situation for bulk water with a continuing decrease of the density towards that of ice I as the temperature is reduced towards the limiting value of $-40^{\circ} \mathrm{C}$ but no confirmation of this behaviour has yet been made. It may be possible to check this feature by temperature-dependent SANS measurements but this experiment is difficult due to the possibility of systematic errors and has not yet been attempted.

\section{Recent studies}

A further series of measurements has been made using a continuous temperature variation rather than a set of measurements at stabilised temperatures. Since the Q-range for significant changes in the $\Delta \mathrm{D}_{\mathrm{M}}(\mathrm{Q}, \Delta \mathrm{T})$ term are restricted to $\mathrm{Q}$-values below $\sim 10 \AA^{-1}$, it is also more convenient to use a diffractometer with a wavelength in the region of $1.3 \AA$ and a high neutron flux. This approach is conveniently achieved by using the rebuilt D20 diffractometer at ILL [11], which is fitted with a $140^{\circ}$ position-sensitive detector. The sample was contained in a $5 \mathrm{~mm}$ diameter vanadium cell, and the temperature was controlled using a ramp with a speed of either 1 or $2 \mathrm{~K} / \mathrm{min}$. Measurements of the whole diffraction pattern up to a Q-value of $6.5 \AA^{-1}$, were made with a typical readout every two minutes. The advantage of this technique is that the datasets are continuously recorded and no time is wasted on temperature stabilisation but there is a minor disadvantage due to the temperature gradient that exists in the sample material throughout the run. Since the changes are systematic with temperature, even near the phase transition points, the $\Delta \mathrm{D}_{\mathrm{M}}(\mathrm{Q}, \Delta \mathrm{T})$ function is well described but the actual mean temperature of the sample is less well defined. A further advantage is that the whole of the diffraction pattern is measured simultaneously so that any change in the relative intensities of ice peaks near a phase transition can be readily observed. Two examples of the new measurements are presented below but there is further analysis to be made on these and other datasets. 


\section{a) Deeply super-cooled water in MCM silicas}

The results for $\mathrm{D}_{2} \mathrm{O}$ in MCM41 silica are shown in the form of stacked $\Delta \mathrm{D}_{\mathrm{M}}(\mathrm{Q}, \Delta \mathrm{T})$ plots in Fig 7 for a cooling run to $220 \mathrm{~K}$ with a reference temperature of $292 \mathrm{~K}$. Several adjacent runs have been added together to give improved statistics. The curves have a constant profile and increase in intensity as the temperature is reduced, confirming the results obtained in the earlier studies. It is also possible to display the data as a contour plot, as given in a preliminary presentation of the measurements at a earlier conference [12]. The full analysis of the data in real-space should improve the accuracy of the previous study and give a more detailed understanding of the way in which the hydrogen-bonded network builds up in this region of deep super-cooling. It should provide a very stringent test of current models of liquid water based on various types of interaction potential as the spatial correlations appear to be very sensitive to the temperature and also extend over a wider $r$ range than is normally considered in computer simulation studies. It seems probable that a full quantum calculation will be required rather than the use of empirical potentials and some possible approaches have already been displayed in other papers presented at this meeting [13].

\section{D20 : MCM : Difference w.r.t. $292 \mathrm{~K}^{\circ}$}

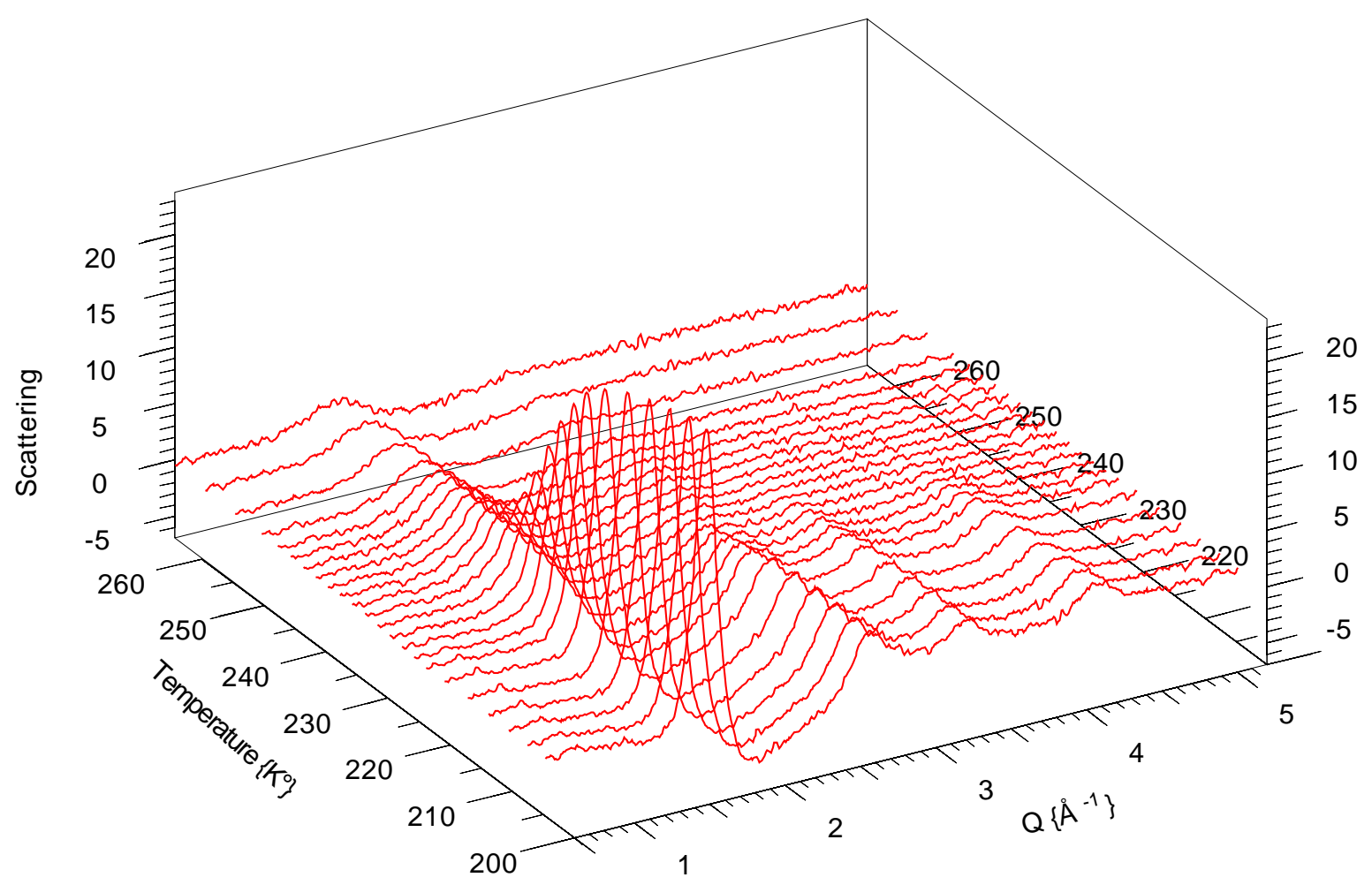

Figure 7. The difference function $\Delta \mathrm{D}_{\mathrm{M}}(\mathrm{Q}, \Delta \mathrm{T})$ for $\mathrm{D}_{2} \mathrm{O}$ water in MCM41 silica taken on the D20 diffractometer as a function of temperature in continuous read-out mode. 


\section{b) Ice melting in larger-pore sol-gel silicas}

Measurements were also made for a sol-gel silica with a $500 \AA$ pore size. In this case, hexagonal ice is formed in the pores even for a partial filling factor of only 0.10 . The good resolution of the D20 instrument and the reduced diffraction broadening effect in the larger pores means that the individual ice triplet is well observed and it is interesting to study the way the profile changes as the temperature approaches the melting temperature on a heating cycle. The results are shown as stacked intensity plots in Fig 8 A close examination of the relative intensities indicates that the central peak reduces more rapidly than the other two peaks as the transition temperature is approached, indicating some premelting effects within $3-5 \mathrm{~K}$ of the phase change. This effect was not expected but presumably indicates that the breakdown of the ordered hydrogen-bond structure of the crystal proceeds by increased vibrational or librational motion with specific characteristics. This phenomenon would be impossible to study in the bulk phase due to the rapid annealing of crystallites and the growth of single domains. It is only because the individual crystallites are isolated within the pore matrix that this behaviour can be identified. Clearly, it will be interesting to make a more detailed investigation using a slower heating rate or for set temperatures as the phenomenon is probably influenced by both time- and temperature-dependent effects.

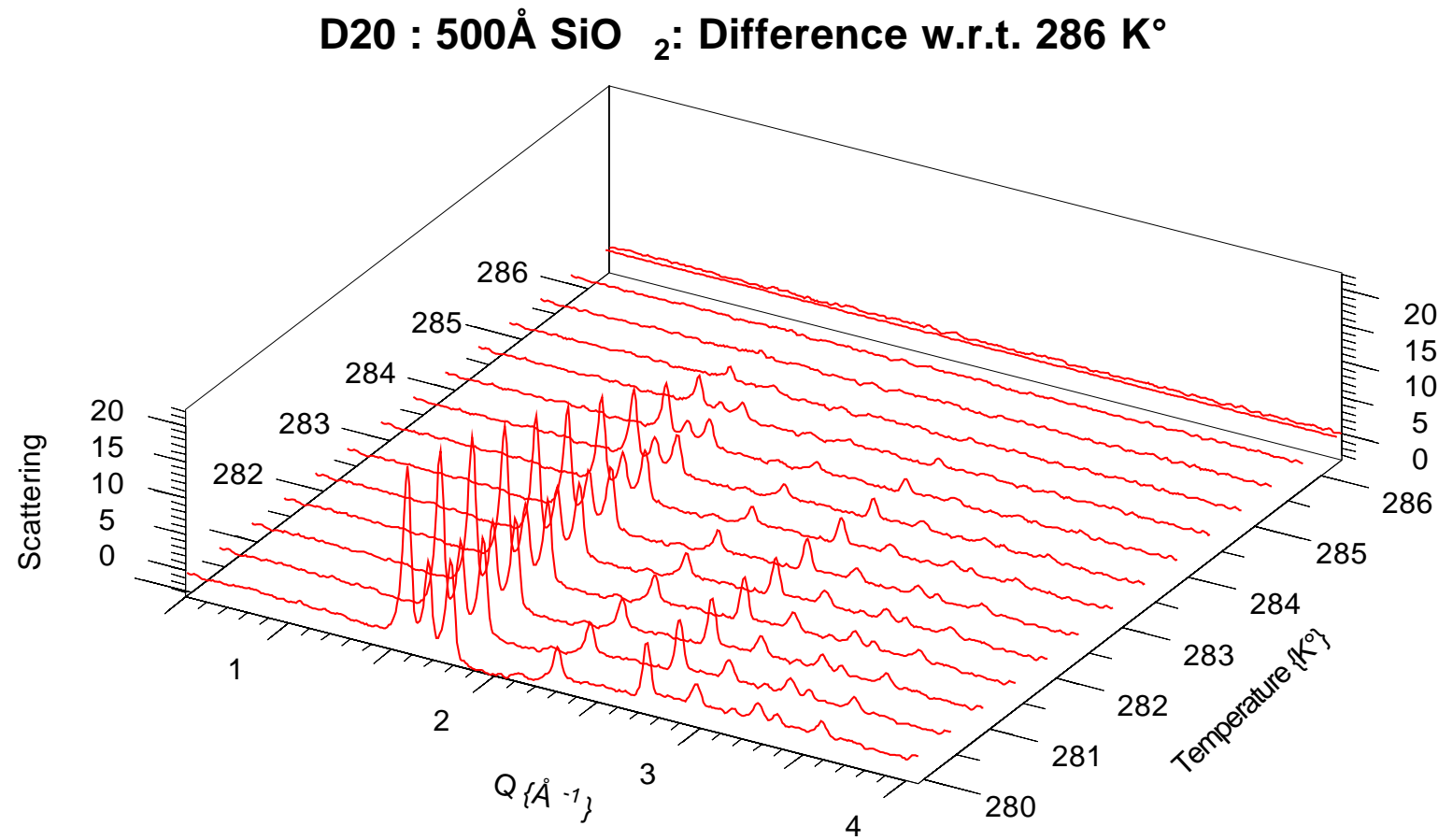

Figure 8. The diffraction pattern for the melting of $\mathrm{D}_{2} \mathrm{O}$ ice in a $500 \AA$ silica with a filling factor of $f=0.1$ as a function of time and temperature; see the text for details

\section{Conclusions and future work}

The previous sections have shown that confined water can exist in the liquid state, at temperatures about $45 \mathrm{~K}$ below the normal freezing point of the bulk liquid. The structure of the deeply super-cooled liquid follows the trends already observed for the temperature variation of the bulk phase, in which there is a continuous evolution towards the open network structure of low-density amorphous ice. Eventually the system undergoes a phase transition to give a solid phase but the crystalline form is 
strongly dependent on the geometrical constraints. For small pores, a defective form of cubic ice is created and there is a complete reversibility across the transition. In the larger pores, hexagonal ice is formed even if the liquid film is quite thin and hysteresis effects are observed.

The picture that emerges from the new datasets [Figs 3a and 3b.] confirms earlier conjectures that the onset of nucleation involves a local fluctuation giving rise to an ice-like nucleus based on tetrahedrally-bonded rings, comprising six molecules. This proto-nucleus is only partially ordered and does not adopt a specific stacking pattern that relates directly to either hexagonal or cubic ice. The diffraction pattern is substantially broadened in the narrow pores of the MCM silicas but probably mimics the pattern seen in the earlier data for the sol-gel silicas with $<100 \AA$ pore size. The profile shown in Fig 8 has not yet been fully explained but presumably arises from crystallites with a high proportion of correlated stacking faults. There is no physical explanation of why this particular form should be the stable (or metastable) state under these conditions but obviously there is a nucleation and growth process that stabilises the crystallites with a finite size determined by the morphology of the silica matrix.

For increased pore sizes, the crystallites can grow larger and it would seem that when a critical size has been reached the subsequent growth is essentially of hexagonal form. The present data suggest that ice formed under normal ambient conditions begins as a defective cubic ice nucleus. This observation is in qualitative agreement with the twophase process seen in the MD computations of Ohmine and colleagues for nucleation of bulk water and also presented at this meeting [14] but a detailed analysis of the developing network structures will need to be compared with the experimental results. Another experimental technique, reported at this meeting [15] uses Mie scattering of laser light to study nucleation in a levitated droplet of $30 \mu$ diameter.

The importance of water/ice in various environments with varying pore morphology and different substrate materials will ensure a continuation of this fascinating subject. Plans have already been made to investigate the behaviour of water/ice in the SBA silicas, which cover an interesting range of $60-100 \AA$ in pore diameter; this range is comparable with many situations involving water channels in bio-membrane systems. Modification of the silica interface can also be made through the grafting of radicals onto the pore walls but it will probably be necessary to check that a high coverage factor is achieved to ensure that unambiguous data are obtained. Another exciting possibility is to investigate the behaviour of water/ice in the very small diameter [5-10 $\AA$ ] pores of carbon nanotubes. Water readily enters into open-ended nanotubes and the vapour-adsorption measurements of Kaneko and colleagues [16] on carbon nanohorns provide the necessary control conditions for conducting this difficult experiment [17]. The diffraction broadening effects will be large and there will be a major contribution from the carbon-water cross terms, so simulation studies will probably be needed to interpret the observations. A new form of ice has already been predicted by Koga et al [18] for the low-temperature solid phase in carbon nanotubes, using MD simulation techniques.

In summary, a clear and consistent qualitative picture of the liquid phase is beginning to emerge from these neutron studies. The temperature difference function is consistent for all the systems studied so far and corresponds to enhanced long-range correlations of the hydrogen-bond network as the temperature is reduced. On 
nucleation, a defective proto-crystallite is formed with a predominantly cubic ice structure but as the crystal size increases, the hydrogen bond network adopts the familiar pattern of hexagonal ice. Although the experimental findings seem incontrovertible, there is still uncertainty about the basic origin of these features and the understanding of water/ice characteristics still presents us with a substantial scientific challenge.

The use of the small pore diameters of the MCM silicas enables the liquid to be routinely cooled to much lower temperatures than previously attained and therefore gives a unique possibility of studying deeply 'super-cooled' water and cubic ice in a stable environment. The use of mesoporous solids therefore provides a convenient 'laboratory' for conducting experimental investigations in conditions that would normally be regarded as highly metastable. The techniques developed here for water/ice are, of course, capable of use for any other molecular liquid and have already been applied to cyclohexane [19].

\section{References}

1) J.G.Powles, Advances in Physics 22, 1 (1973)

2) I.P.Gibson and J.C.Dore, Mol Phys. 481019 (1983)

3) D.C.Steytler, J.C.Dore and C.J.Wright, Mol Phys 481031 (1983)

4) D.C.Steytler, J.C.Dore and C.J.Wright, Mol Phys 561001 (1985)

5) J.C.Dore, Chem Phys 258327 (2000)

6) M.C.Bellissent-Funel et al, Europhys Letts 2241

7) D.Blakey, PhD Thesis, University of Kent (1994) and M.R.Choudhury, J.C.Dore and J.T.Wenzel, J.Non.Cryst Solids 53247 (1982)

8) J.M.Baker, J.C.Dore and P.Behrens, J.Phys.Chem B101 6226 (1997)

9) The theoretical formalism was originally described in the interpretation of diffraction data for carbon blacks and is often referred to as 'the Warren profile'; B.E.Warren Phys. Rev. 59693 (1941).

The application to the cubic ice profiles is discussed in J.M.Baker, $\mathrm{PhD}$

10) G.Findenegg, private communication and Phys. Chem. Chem Phys., 3, 11851195 (2001).

11) The new D20 diffractometer is described in the instrument section of the ILL website; www.ill.fr

12) J.Dore, B.Webber, M.Hartl, P.Behrens and T.Hansen, Physica A314 501 (2002)

13) S.S.Xanthias, this volume

14) M.Matsumoto, S.Saito and I.Ohmine, Nature 416409 (2002) and also I. Ohmine, this volume

15) P.Stoeckel, J.Klein, I. Weidinger, H.Baumgartel and T.Leisner, see also H.Baumgartel in this volume

16) K.Kaneko, private communication and E.Bekyrova et al, Chem Phys Letts 366 463 (2002).

17) An experimental study of water/ice in carbon nanohorns by neutron diffraction is feasible and has been proposed; J.C.Dore, A.Burian and K.Kaneko; currently under consideration (2002-03)

18) K.Koga, G.T.Gao,H. Tanaka and X.C.Zeng, Nature 412802 (2001)

19) H.Farman, J.C.Dore and J.B.W.Webber, J.Mol.Liq. 96357 (2002) 